\section{Novel aptamer to von Willebrand factor A1 domain (TAGX-0004) shows total inhibition of thrombus formation superior to ARC1779 and comparable to caplacizumab}

\author{
Kazuya Sakai, ${ }^{1}$ Tatsuhiko Someya, ${ }^{2}$ Kaori Harada, ${ }^{2}$ Hideo Yagi, ${ }^{1}$ Taei Matsui ${ }^{3}$ \\ and Masanori Matsumoto ${ }^{1}$ \\ ${ }^{1}$ Department of Blood Transfusion Medicine, Nara Medical University, Kashihara and \\ ${ }^{2}$ TAGCyx Biotechnologies Inc, Tokyo and ${ }^{3}$ Clinical Laboratory Medicine, Fujita Health \\ University School of Medical Sciences, Toyoake, Japan
}

\section{ABSTRACT}

$\mathrm{V}$ on Willebrand factor (VWF) is a blood glycoprotein that plays an important role in platelet thrombus formation through interaction between its A1 domain and platelet glycoprotein Ib. ARC1779, an aptamer to the VWF A1 domain, was evaluated in a clinical trial for acquired thrombotic thrombocytopenic purpura (aTTP). Subsequently, caplacizumab, an anti-VWF A1 domain nanobody, was approved for aTTP in Europe and the USA. We recently developed a novel DNA aptamer, TAGX-0004, to the VWF A1 domain; it contains an artificial base and demonstrates high affinity for VWF. To compare the effects of these three agents on VWF A1, their ability to inhibit ristocetin- or botrocetin-induced platelet aggregation under static conditions was analyzed, and the inhibition of thrombus formation under high shear stress was investigated in a microchip flow chamber system. In both assays, TAGX-0004 showed stronger inhibition than ARC1779, and had comparable inhibitory effects to caplacizumab. The binding sites of TAGX-0004 and ARC1779 were analyzed with surface plasmon resonance performed using alanine scanning mutagenesis of the VWF A1 domain. An electrophoretic mobility shift assay showed that R1395 and R1399 in the A1 domain bound to both aptamers. R1287, K1362, and R1392 contributed to ARC1779 binding, and F1366 was essential for TAGX-0004 binding. Surface plasmon resonance analysis of the binding sites of caplacizumab identified five amino acids in the VWF A1 domain (K1362, R1392, R1395, R1399, and K1406). These results suggest that TAGX-0004 possesses better pharmacological properties than caplacizumab in vitro and might be similarly promising for aTTP treatment.

\section{Introduction}

Von Willebrand factor (VWF) is a large multimeric plasma glycoprotein that plays an essential role in tethering circulating platelets to damaged endothelial cells. ${ }^{1}$ Interaction between the VWF A1 domain and the platelet glycoprotein (GP) Ib receptor leads to rapid development of VWF-rich platelet thrombi, thus preventing further bleeding. ${ }^{2}$ Although normal amounts of VWF are usually helpful in hemostasis reactions, several VWF-mediated diseases, such as acute coronary syndrome ${ }^{3,4}$ cerebral infarction, ${ }^{5}$ and thrombotic thrombocytopenic purpura (TTP), ${ }^{6}$ demonstrate remarkably elevated plasma levels of VWF. Inhibiting VWF activity by blocking its A1 domain has recently been demonstrated to be an attractive therapeutic target in these arterial thromboses. ${ }^{7-10}$

Caplacizumab (Ablynx), an anti-VWF A1 humanized nanobody, ${ }^{11,12}$ was approved by the European Medicines Agency in 2018 and by the US Food and Drug Administration in 2019 for the treatment of acquired TTP (aTTP). The antiVWF A1 aptamer ARC1779 (Archemix) was developed as an antithrombotic agent for use in patients with aTTP, and it showed sufficient inhibitory effects on VWF activity without any severe bleeding events in a randomized control trial. ${ }^{13}$ However, ARC1779 has not yet been approved for the treatment of VWF-mediated
Ferrata Storti Foundation
Haematologica 2020
Volume 105(11):2631-2638

\section{Correspondence:}

MASANORI MATSUMOTO

mmatsumo@naramed-u.ac.jp

Received: August 16, 2019.

Accepted: December 18, 2019.

Pre-published: December 19, 2019.

doi:10.3324/haematol.2019.235549

(C)2020 Ferrata Storti Foundation

Material published in Haematologica is covered by copyright. All rights are reserved to the Ferrata Storti Foundation. Use of published material is allowed under the following terms and conditions:

https://creativecommons.org/licenses/by-nc/4.0/legalcode. Copies of published material are allowed for personal or internal use. Sharing published material for non-commercial purposes is subject to the following conditions: https://creativecommons.org/licenses/by-nc/4.0/legalcode, sect. 3. Reproducing and sharing published material for commercial purposes is not allowed without permission in writing from the publisher. 
thrombosis. We recently generated a novel DNA aptamer, TAGX-0004, that contains the artificial hydrophobic base 7-(2-thienyl)imidazo[4,5-b]pyridine (Ds); this aptamer targets human VWF A1 with very high affinity $\left(\mathrm{K}_{\mathrm{D}}=61.3\right.$ pM) and specificity. ${ }^{14}$

In this study, we compared TAGX-0004, ARC1779, and caplacizumab in terms of their inhibitory effects on VWF activity, by performing conventional platelet aggregation assays, ristocetin- and botrocetin-induced platelet aggregation assays (RIPA and BIPA, respectively), and shear-stressinduced aggregation assays using the microchip flow chamber system $\left(\mathrm{T}^{-} \mathrm{TAS}{ }^{\circledR}\right){ }^{15}$ To assess the affinity and binding sites of the two aptamers, we performed biophysical interaction analysis and alanine scanning mutagenesis of the VWF A1 domain. In addition, we analyzed the binding sites of caplacizumab using surface plasmon resonance (SPR).

\section{Methods}

Details of the materials and methods are presented in the Online Supplementary Materials and Methods section.

\section{Sources of nucleoside, oligonucleotides, nanobody, and protein}

The artificial nucleoside phosphoramidite (dDs-CE Phosphoramidite) was synthesized as described previously. ${ }^{16,17}$ The oligonucleotides TAGX-0004 and ARC1779 (without polyethylene glycol [PEG]) were synthesized by and purchased from GeneDesign, Inc. (Osaka, Japan). The oligonucleotide sequence of TAGX-0004 (as Rn-DsDs-51mh2) 14 $^{14}$ and that of ARC1779 $9^{18}$ were reported previously. The anti-human VWF nanobody caplacizumab (as TAB-234) was purchased from Creative-Biolabs (New York, NY, USA). The recombinant human VWF A1 domain protein was purchased from U-Protein Express BV (Utrecht, the Netherlands), and was used for the measurement of the binding affinity to antihuman VWF A1 agents using SPR.

\section{Alanine-scanning mutagenesis}

Based on previous reports that described the interaction between VWF A1 and GPIb, ${ }^{19}$ botrocetin ${ }^{20}$ or ARC1172, ${ }^{21}$ we designed 16 alanine-substituted mutants of the human VWF A1 domain (R1287, K1312, R1334, R1336, K1348, K1362, F1366, K1371, E1376, R1392, R1395, R1399, K1406, K1423, R1426, and K1430). These mutant proteins were generated using a cell-free expression system (Taiyo Nippon Sanso Corporation, Tokyo, Japan).

\section{Platelet aggregation test}

A platelet aggregation test (PAT) was performed with PRP313M aggregometer (TAIYO Instruments INC, Osaka, Japan). Aggregation inducing substances and their final concentration were as follows; ristocetin $(1.5 \mathrm{mg} / \mathrm{mL})$, botrocetin $(1.0 \mu \mathrm{g} / \mathrm{mL})$, collagen $(4 \mu \mathrm{g} / \mathrm{mL})$, epinephrine $\left(1.0 \times 10^{-4} \mathrm{M}\right)$ and adenosine diphosphate (ADP) $\left(1.0 \times 10^{-5} \mathrm{M}\right)$.

\section{Total thrombus formation analysis system}

The total thrombus formation analysis system (T-TAS ${ }^{\circledR}$ ) (Zacros, Fujimori Kogyo Co. Ltd., Tokyo, Japan) is a micro-chip flow-chamber device used to visually and quantitatively analyze thrombus formation in whole blood samples under various blood flow conditions. ${ }^{15}$ Whole blood samples with each anti-VWF A1 agent were applied onto a collagen I coated micro-chip (PL chip). Subsequently, the thrombus formation in the capillaries and an increase in flow pressure were observed.

\section{Electrophoresis mobility shift assay}

The binding abilities of the two aptamers to the human VWF A1 domain and its alanine-substituted mutants were analyzed by electrophoresis mobility shift assay (EMSA). Each aptamer (final concentration of $100 \mathrm{nM}$ ) was mixed with VWF A1 (final concentration of $0-800 \mathrm{nM}$ ) in binding buffer and incubated at $37^{\circ} \mathrm{C}$ for 30 minutes ( $\mathrm{min}$ ), then subjected to $8 \%$ native PAGE in $0.5 \times \mathrm{TBE}$ buffer for $50 \mathrm{~min}$ at room temperature $(200 \mathrm{~V} / \mathrm{cm})$. The aptamerVWF A1 complexes were detected as a shift band, and the band patterns were detected by SYBR Gold. The dissociation rate (KD) was calculated by Scatchard plot analysis.

\section{Surface plasmon resonance}

Competition assays with the two aptamers were performed by surface plasmon resonance (SPR) analysis using Biacore T200 (GE Healthcare UK Ltd., Little Chalfont, UK), as described previously. ${ }^{14}$

We also performed SPR analysis to investigate the binding site of the VWF A1 domain to caplacizumab.

\section{Structure models of the VWF A1 domain}

Three-dimensional (3D) structure models of the VWF A1 domain were visualized with the PyMOL Molecular Graphics System (DeLano Scientific, San Carlos, CA, USA).

\section{Ethical statement}

This study was approved by the ethics committee of Nara Medical University. Written informed consent was obtained from the individual who donated plasma for the use in this study.

\section{Results}

Inhibitory effects on platelet aggregation

The inhibitory effects of TAGX-0004, ARC1779, and caplacizumab under static conditions were analyzed by PAT. Figure 1 shows representative results of the analyses of the three types of plasma. All three agents showed inhibition activities in both RIPA and BIPA. However, the inhibitory effects on platelet aggregation differed between the three agents. In RIPA, the $80 \%$ maximal inhibitory concentration $\left(\mathrm{IC}_{80}\right)$ of TAGX-0004 was $50 \mathrm{nM}$, whereas that of ARC1779 was $500 \mathrm{nM}$ and that of caplacizumab was $50 \mathrm{nM}$; thus TAGX-0004 blocked VWF function via the A1 domain approximately 10 times more potently than ARC1779, and exhibited a similar potency as caplacizumab. In BIPA, the $\mathrm{IC}_{80}$ of TAGX-0004 was $50 \mathrm{nM}$, whereas that of ARC1779 was $500 \mathrm{nM}$ and that of caplacizumab was $50 \mathrm{nM}$, respectively; thus TAGX-0004 blocked VWF function approximately 10 times more potently than ARC1779 showing a similar potency as caplacizumab. None of the three agents inhibited platelet aggregation induced by collagen, epinephrine, or ADP (data not shown).

\section{Inhibitory effects on thrombus formation}

To assess the inhibitory effect of the three agents on platelet thrombus formation, we performed T-TAS. In this study, complete inhibition was defined as an increase in flow pressure from baseline of no more than $10 \mathrm{kPa}$. TAGX-0004, ARC1779, and caplacizumab were analyzed three times each, and Figure 2 shows representative flow pressure curves. Both TAGX-0004 and caplacizumab prevented thrombus occlusion under the flow condition at 50 nM. In contrast, ARC1779 did not demonstrate complete inhibition, even at a concentration of 1,000 nM. These 
TAGX-0004

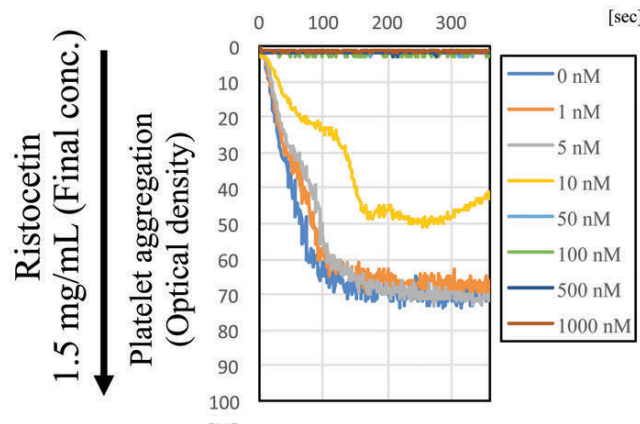

[sec]

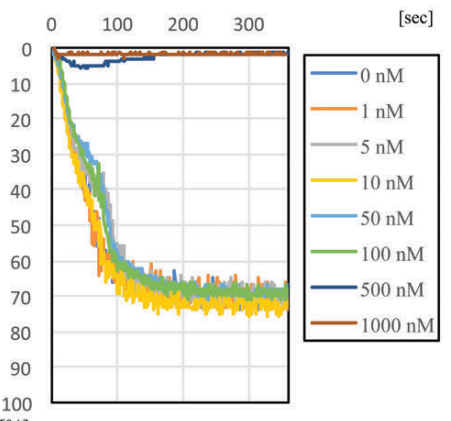

[\%]
ARC1779

Caplacizumab
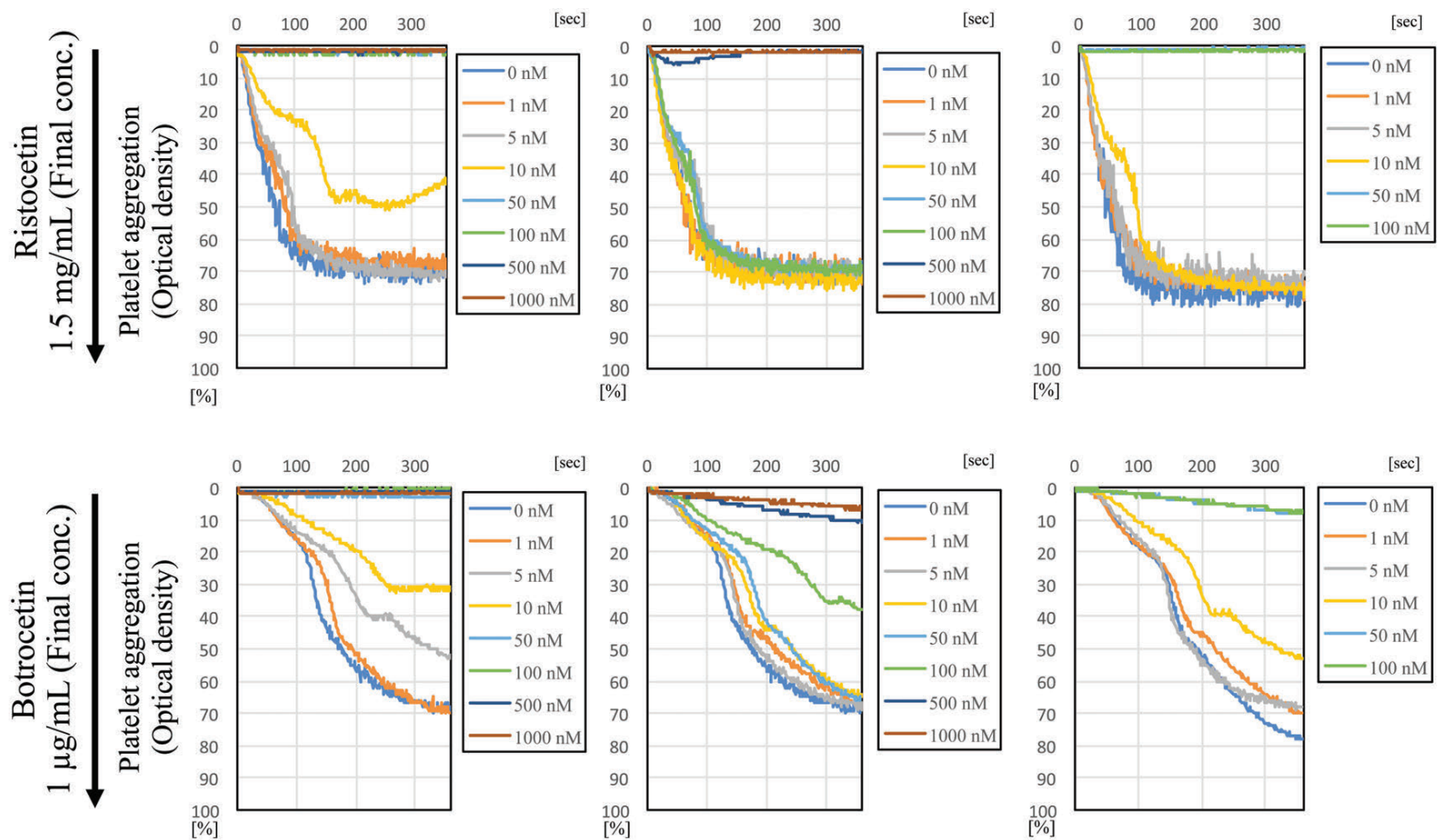

$[\%]$

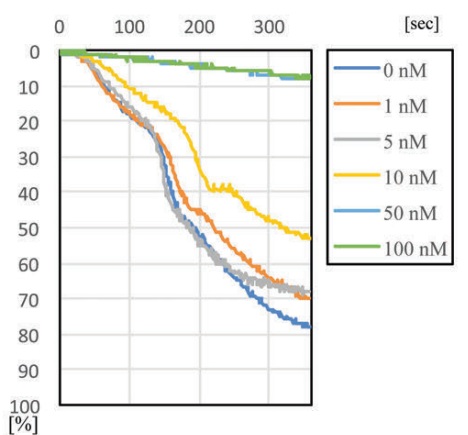

Figure 1. The inhibitory effects of TAGX-0004, ARC1779, and caplacizumab on platelet aggregation under static conditions. As shown in the upper left panel, TAGX0004 completely inhibited ristocetin-induced platelet aggregation (RIPA) at a concentration of $50 \mathrm{nM}$. In contrast, the concentration required for ARC1779 was 500 $\mathrm{nM}$, and that for caplacizumb was $50 \mathrm{nM}$. As shown in the lower left panel, TAGX-0004 inhibited botrocetin-induced platelet aggregation (BIPA) at a concentration of $50 \mathrm{nM}$. The concentration required for ARC1779 was $500 \mathrm{nM}$, and that for caplacizumab was $50 \mathrm{nM}$.

results indicate that TAGX-0004 inhibits platelet thrombus formation at least 20 times more potently than ARC1779 under high shear stress, and with a similar potency as caplacizumab.

\section{Biophysical interaction analysis with EMSA}

Biophysical interaction analysis with EMSA was performed four times for each aptamer. Figure 3 shows that the affinity of TAGX-0004 to VWF A1 domain $\left(K_{D}=2.2 \pm\right.$ $0.9 \mathrm{nM}[\mathrm{n}=4])$ was approximately 16 -fold higher than that of ARC1779 $\left(K_{D}=35.5 \pm 1.5 \mathrm{nM}[\mathrm{n}=4]\right)$.

\section{Alanine scanning mutagenesis with EMSA}

Figure 4 shows the results of EMSA using 16 alaninemutated VWF A1 with TAGX-0004 (A) or ARC1779 (B). First, we analyzed nine mutants as shown in the left panels. Based on the results of this initial analysis, we then analyzed additional seven mutants as shown in the right panels. Of these, 10 VWF A1 mutants (K1312A, R1334A, R1336A, K1348A, K1371A, E1376A, K1406A, K1423A, $\mathrm{R} 1426 \mathrm{~A}$, and $\mathrm{K} 1430 \mathrm{~A})$ formed shifted bands representing complexes between an aptamer and VWF A1, as well as between wild-type (WT) VWF A1 and each aptamer. In the remaining six mutants, the intensity of the complexed bands was decreased, and the intensity of the unbound aptamer bands was increased. Of these mutants, R1395A and R1399A showed decreased intensity in both TAGX0004 and ARC1779 band complexes.

While the binding affinity to ARC1779 was significantly decreased in R1287A, K1362A, and R1392A, the binding affinity to TAGX-0004 was decreased in F1366A. These findings suggest that R1395 and R1399 are essential residues for binding to both aptamers, and that R1287, K1362, and R1392 probably contribute to binding to only ARC1779, while F1366 is required for binding to TAGX-0004. Our data indicate that both aptamers bind to the VWF A1 domain, even though the residues necessary for this binding are partially different, and these differences might affect their VWF inhibitory activity.

\section{Competition assay with Biacore}

To compare the binding sites of the aptamers to the VWF A1 domain, a competition assay was performed by SPR using Biacore. Biotinylated TAGX-0004 was immobilized on the sensor chip SA. Then, VWF A1 and a competitor aptamer (TAGX-0004 or ARC1779) as an analyte was injected onto the chip. The results of a self-competition assay using TAGX-0004 showed that the amount of VWF A1 binding to the chip decreased depending on the concentration of the competitor TAGX-0004 (Online Supplementary Figure S1). ARC1779 also dose-dependently decreased the response unit value. These results indicate that TAGX-0004 and ARC1779 bind to the overlapped region on the VWF A1 domain. Moreover, the competitive ability of ARC1779 against immobilized TAGX-0004 on the chip surface was lower than that of TAGX-0004 against TAGX-0004. These results were consistent with those of EMSA and indicated that the binding affinity of TAGX-0004 for VWF A1 was higher than that of ARC1779. 
VWF A1 domain binding site of caplacizumab

We performed SPR analysis to investigate the VWF A1 domain binding site of caplacizumab. After immobilizing caplacizumab on the sensor chip CM5, 16 alanine-substituted mutants of VWF A1 were analyzed (Figure 5). The relative binding amount of WT was defined as $100 \%$. The binding amount of each mutant was expressed as the relative ratio compared to that of WT. When a relative ratio of $80 \%$ was set as the cut-off, five mutants were judged as positive. These results indicate that these five residues (K1362, R1392, R1395, R1399, and K1406) are likely the binding sites of caplacizumab.

\section{Comparison of VWF A1 domain binding sites of TAGX-0004, ARC1779, and caplacizumab}

Figure 6 shows 3D structure models of the VWF A1 domain. The amino acid residues shown in red indicate the essential residues for binding to each aptamer or capla- cizumab. The residues shown in yellow are not involved in binding. Of the 16 alanine-substituted VWF A1 mutants, EMSA predicted that three amino acids (F1366, R1395, and R1399) are binding sites for TAGX-0004, as shown in red in Figure 6 on the left. The remaining 13 amino acid residues are shown in yellow. As for ARC1779, five amino acid residues (R1287, K1362, R1392, R1395, and R1399) play an important role in binding to VWF A1, as shown in red in Figure 6 in the middle. Of these, R1395 and R1399 are amino acids shared by both aptamers. Further, using Biacore we identified five amino acid residues in the VWF A1 domain (K1362, R1392, $\mathrm{R} 1395, \mathrm{R} 1399$, and K1406) that are binding sites to caplacizumab, as shown in red in Figure 6 on the right. Of these, K1406 is unique to caplacizumab. The differences in VWF A1 domain binding sites suggest that these aptamers or nanobody might have unique effects on platelet aggregation and thrombosis formation.

\section{TAGX-0004}
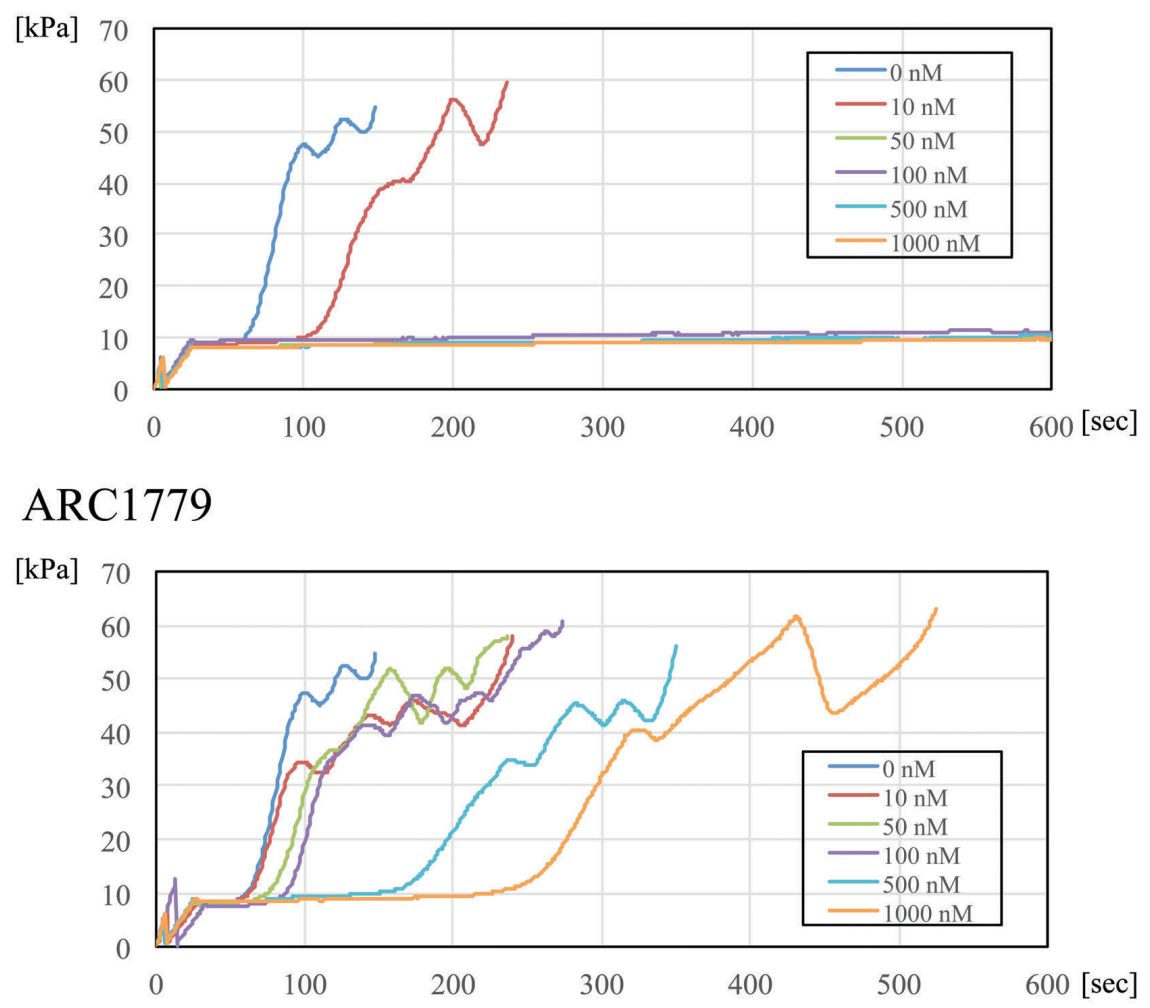

\section{Caplacizumab}

$[\mathrm{kPa}]$

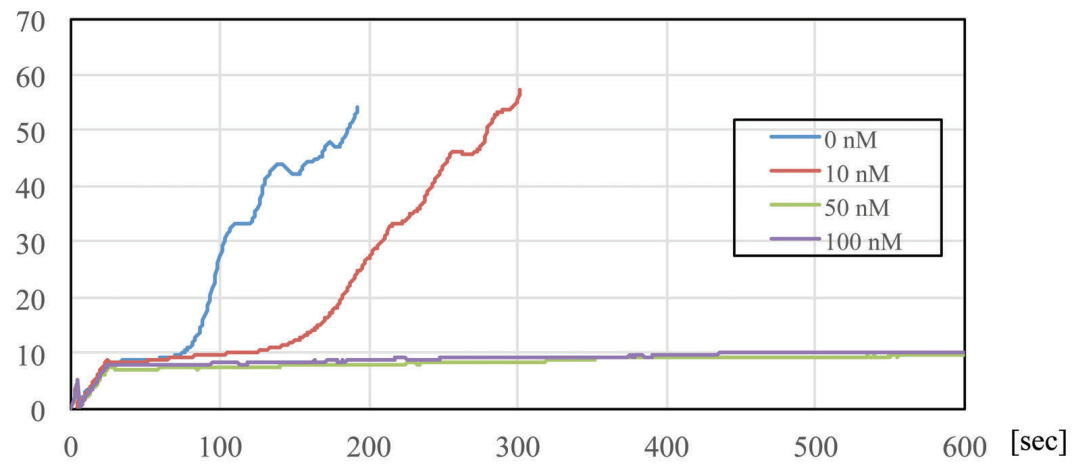

Figure 2. The inhibitory effects of TAGX-0004, ARC1779, and caplacizumab on platelet thrombus formation under high shear stress. Pressure curves at various concentrations of each agent are shown; curves end at the point of complete microcapillary occlusion. As shown in the upper panel, TAGX-0004 inhibited thrombus formation under high shear conditions at a final concentration of $50 \mathrm{nM}$. The middle panel shows that ARC1779 did not achieve complete inhibition even at a concentration of $1,000 \mathrm{nM}$. The lower panel shows that caplacizumab completely inhibited thrombus formation at a concentration of $50 \mathrm{nM}$. 


\section{Discussion}

VWF plays a pivotal role in the initial phase of platelet thrombus formation under high shear stress through the interaction between its A1 domain and platelet GPIb. ${ }^{1,2}$ The binding site of the VWF A1 domain is usually cryptic and prevents spontaneous binding to platelets. High shear stress of blood flow induces conformational changes in VWF and exposes the VWF A1 domain. Therefore, the association between VWF A1 and platelet GPIb usually occurs under high shear stress. ${ }^{2}$

Inhibition of VWF A1 domain binding to platelet GPIb can potentially prevent the development of platelet thrombus formation that causes cardiac infarction, cerebral infarction, and TTP. TTP results from the formation of platelet thrombi in the microvasculature due to a deficiency of ADAMTS13, a VWF-cleaving protease..$^{22,23}$ Therefore, preventing VWF A1 binding to platelet GPIb could be a promising therapeutic target for TTP. For this purpose, caplacizumab was evaluated for its anti-thrombotic effects in a phase II clinical trial (the TITAN study) ${ }^{11}$ and a phase III clinical trial (the HERCULES study). ${ }^{12}$ These studies reported a therapeutic effect of caplacizumab on aTTP, including faster recovery of platelet counts, fewer plasma exchange sessions, and shorter hospital stays. A bleeding event was reported as a common adverse event in patients treated with caplacizumab compared to patients without it. ${ }^{12}$ In the TITAN and HERCULES study, 36 and 72 patients of aTTP were treated with caplacizum$\mathrm{ab}$ and $19(54 \%)$ and $46(65 \%)$ patients had bleeding events, respectively. The most common adverse events were epistaxis and gingival bleeding, neither of which generally required treatment.

Nucleic acid aptamers are single-strand DNA or RNA molecules that can form $3 \mathrm{D}$ structures capable of specifically binding to proteins or other cellular targets. They are superior to existing antibody products in terms of their specificity, manufacturing cost, relatively small size, and non-immunogenicity. Pegaptanib (Macugen ${ }^{\circledR}$, Pfizer) is the only aptamer approved by the Food and Drug Adminstration in 2004 for the treatment of wet age-relat- ed macular degeneration blocking vascular endothelial growth factor. ${ }^{24}$ As of June 2019, no aptamer for the patients with coagulation or thrombotic disorder has been approved. However, some aptamers targeting coagulation factors, ARC 1779 against VWF A1, NU172 (ARCA Biopharma) against factor IIa, and REG1/REG2 (Regado Biosciences) against factor IXa, stepped into clinical trials. ${ }^{13,25-27}$ ARC1779 was developed to target the VWF A1 domain and was evaluated in a phase II clinical trial in patients with aTTP. ${ }^{13}$ Unfortunately, the recruitment of patients in this trial was terminated without completing the study due to sponsor-related financial issues, but seven patients with aTTP received combined therapy with intravenous ARC1779 injections and plasma exchange. ${ }^{13}$ The trial showed no severe adverse events such as bleeding, even in patients with aTTP who had severe thrombocytopenia. ${ }^{13}$ A study of healthy volunteers confirmed that this aptamer had an antithrombotic effect and did not cause severe bleeding events. ${ }^{28}$

TAGX-0004 is a novel DNA aptamer that targets the VWF A1 domain and contains Ds, an artificial nucleic acid. TAGX-0004 was obtained using modified SELEX (systemic evolution of ligands by exponential enrichment) methods incorporating the Ds base. ${ }^{14}$ Since the Ds base has no complementary base in nature, Ds-containing DNA aptamers can have unique 3D structures. The high hydrophobicity of the Ds base may contribute to significantly high binding affinity to target proteins. In fact, the results of EMSA showed that the TAGX-0004 binding affinity for VWF $\left(\mathrm{K}_{\mathrm{D}}=2.2 \pm 0.9 \mathrm{nM}\right)$ is 16-fold higher than that of ARC1779 $\left(\mathrm{K}_{\mathrm{D}}=35.5 \pm 1.5 \mathrm{nM}\right)$ (Figure 3). However, the $K_{D}$ values in this study might be underestimated in comparison with previous reports; the $\mathrm{K}_{\mathrm{D}}$ value of TAGX0004 was shown to be 61.3 pM by a SPR assay, ${ }^{14}$ and the $\mathrm{KD}$ value of ARC1779 was $2 \mathrm{nM}$ in a RI-labeled assay. ${ }^{18}$ Previous results also indicated that the binding affinity of TAGX-0004 was much higher than that of ARC1779.

The binding affinity of aptamers to VWF A1 domain might affect the interaction between VWF and platelets. Although both TAGX-0004 and ARC1779 inhibited the platelet aggregations which were induced by ristocetin

\section{A TAGX-0004}

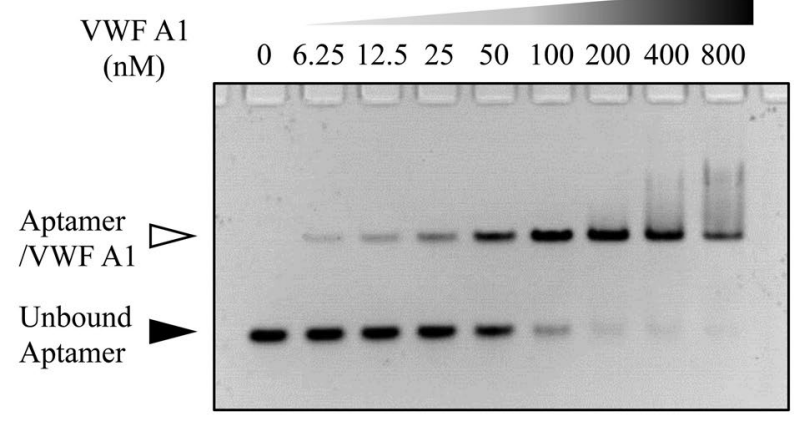

$\mathrm{K}_{\mathrm{D}}=2.2 \pm 0.9 \mathrm{nM}$

\section{B ARC1779}
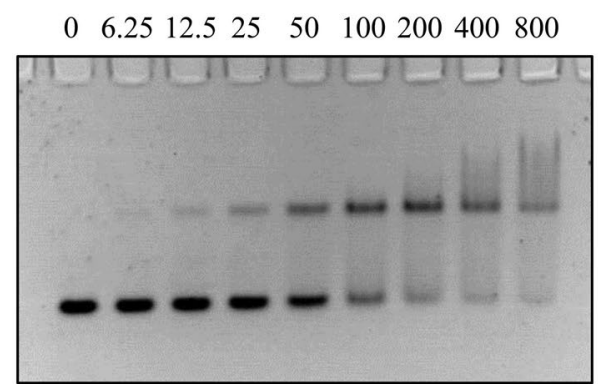

$\mathrm{K}_{\mathrm{D}}=35.5 \pm 1.5 \mathrm{nM}$

Figure 3. Biophysical interaction analysis of TAGX-0004 and ARC1779 with electrophoresis mobility shift assay (EMSA). The white arrowhead indicates the complex of wild-type (WT) recombinant von Willebrand factor (VWF) A1 with an aptamer (TAGX-0004 or ARC1779). The black arrowhead indicates unbound aptamer. Increasing the concentration of WT VWF A1 increased the density of complex bands and decreased that of unbound aptamer bands. These experiments were performed four times for each aptamer. The dissociation rates (KD) of TAGX-0004 and ARC1779 were 2.2 $\pm 0.9 \mathrm{nM}$ and $35.5 \pm 1.5 \mathrm{nM}$, respectively. 


\section{A TAGX-0004}

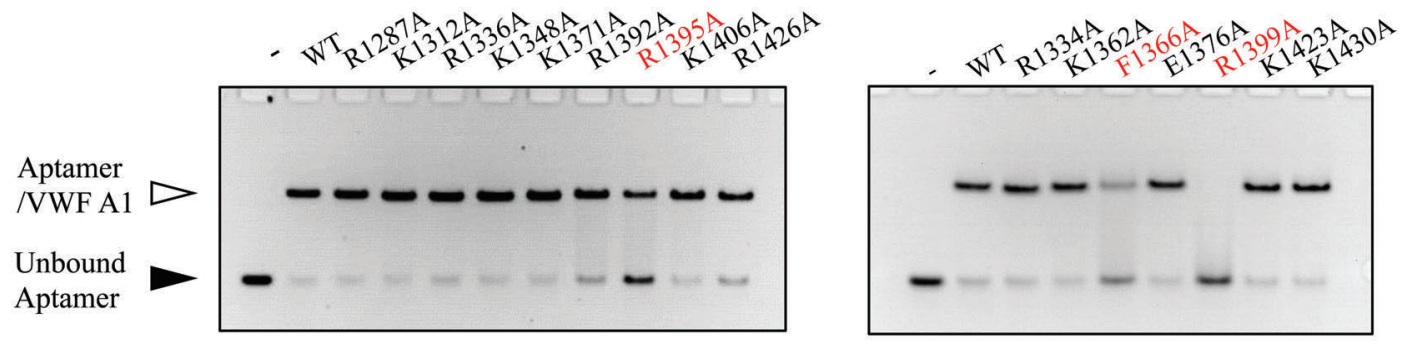

B ARC1779
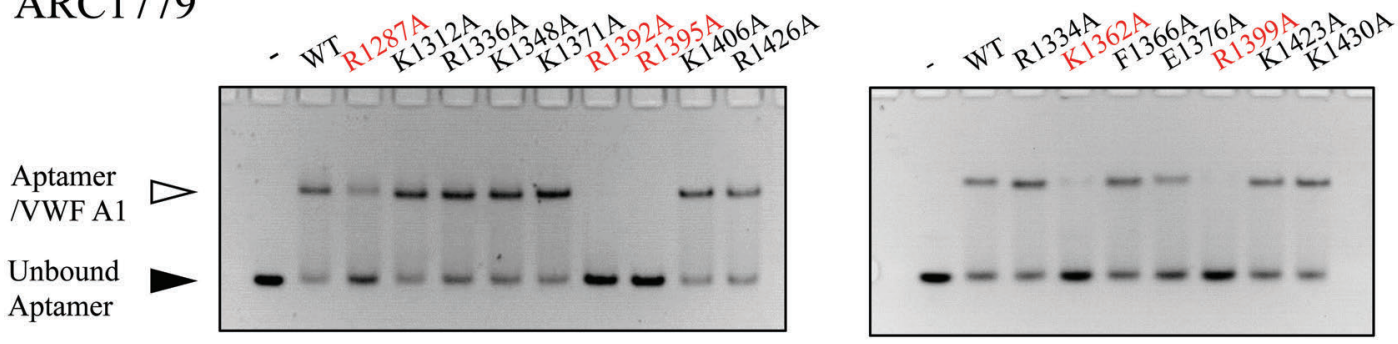

Figure 4. Electrophoresis mobility shift assay (EMSA) using alanine-scanning mutants for analyzing von Willebrand factor A1 binding to TAGX-0004 and ARC1779 The white arrowhead indicates the complex of von Willebrand factor (VWF) A1 with an aptamer (TAGX-0004 or ARC1779). The black arrowhead indicates unbound aptamer. The leftmost lane in each gel (-) represents aptamer only, without a VWF A1 mutant. Using both aptamers, 10 VWF A1 mutants (K1312A, R1334A, R1336A, K1348A, K1371A, E1376A, K1406A, K1423A, R1426, and K1430A) formed complexes with aptamers, as did wild-type (WT) VWF A1. With TAGX-0004, as shown in the top panels, three mutants (F1366A, R1395A, and R1399A, indicated in red letters) demonstrated decreased densities of complex bands and increased densities of unbound bands, indicating that these amino acids were important for the binding of VWF A1 to TAGX-0004. With ARC1779, as shown in the lower panels, five mutants (R1287A, K1362A, R1392A, R1395A, and R1399A indicated in red letters) demonstrated decreased densities of complex bands and increased densities of unbound bands.

and botrocetin, the minimum concentration of TAGX0004 necessary for inhibition is significantly lower than that of ARC1779 in both RIPA and BIPA (Figure 1). PAT analysis revealed that TAGX-0004 can block VWF function via the A1 domain at least 10 times more strongly than ARC1779. T-TAS also revealed that TAGX-0004 is superior to ARC1779 at inhibiting VWF function (Figure 2). In addition, compared with caplacizumab, TAGX-0004 showed equally effective inhibition against thrombosis formation under various blood flow conditions. Moreover, TAGX-0004 has a unique mini-hairpin DNA structure that offers benefits in pharmaceutical applications, ${ }^{14}$ specifically by conferring resistance to degradation by nucleases. This structure should extend the half-life of this molecule in vivo.

As described above regarding the adverse effects of caplacizumab treatment, there are still concerns regarding bleeding caused by anti-VWF antagonists. Although antiVWF agents have demonstrated superior safety profiles in this regard compared to anti-platelet agents, measures to quickly treat bleeding that occurs during aptamer treatment should be prepared. We speculate that if a neutralizing agent comprising a DNA sequence that is partially complementary to that of a specific aptamer is prepared, it may serve as an effective antidote as introduced in the literature. ${ }^{29}$ In the case of TAGX-0004, such a partial DNA sequence may contain pyrrole-2-carbaldehyde $(\mathrm{Pa})$, the complementary base pair to Ds. ${ }^{16}$ In the past development of antithrombotic aptamer therapeutics, similar approaches of antidotes were taken to neutralize the unwanted effect of an aptamer, even though such aptamers are still to be approved. ${ }^{26}$ The specific antidote could contribute to control severe bleeding compared to caplacizumab.

Alanine mutagenesis analysis of the VWF A1 domain was performed in this study to determine the binding sites of TAGX-0004, ARC1779, and caplacizumab. The binding sites of ARC1779, but not TAGX-0004 or caplacizumab, were reported previously. ${ }^{21}$ Our results show that the binding sites of these three agents only partially correspond to each other (Figure 6). Huang et al. ${ }^{21}$ reported that 18 amino acid residues in the VWF A1 domain were binding sites for ARC1172, which has the same fundamental structure as ARC1779. That study did not identify R1399 as a binding site, even though in the present study it is a common binding site for all three agents. Chen et al. identified R1399 as one of the key amino acids for the effect of VWF to hemostasis and thrombosis. ${ }^{30}$ Interestingly, a hydrophobic amino acid residue (F1366) is required to bind to TAGX-0004, which has two hydrophobic Ds bases. Matsunaga et al. previously reported that the number of Ds bases (0-2) strongly correlated with binding affinity to VWF $A 1^{14}$ and we confirmed that Ds-free TAGX-0004 failed to inhibit thrombus formation on T-TAS (data not shown). These results indicate that the base of Ds interacts directly with the VWF A1 domain and therefore F1366 appears to be an essential and unique binding site of TAGX-0004 to the VWF-A1 domain with hydrophobic interaction (e.g., pi-stacking). Of note, alanine scanning of caplacizumab showed that like ARC1779 and TAGX-0004, both R1395 and R1399 are nec- 
essary to bind the VWF A1 domain (Figure 6).

Due to the method limitations, the affinity between the VWF A1 domain and TAGX-0004 or ARC1779 was not analyzed by the SPR method. Since both oligonucleotides and the sensor chip are negatively charged, the $K_{D}$ value was underestimated when we immobilized the VWF A1 protein on the sensor chip and applied TAGX-0004 or ARC1779 as an analyte. Therefore, we performed alanine scanning by EMSA for DNA aptamers. In addition, the binding site of caplacizumab to VWF A1 domain was not analyzed with EMSA, which was used to assess TAGX0004 and ARC1779, since this technique is not suitable for the analysis of proteins such as nanobodies and antibodies. Secondly, ARC1779 was originally PEGylated. However, we used ARC1779 without PEG in this study because we found no obvious differences between ARC1779 with and without PEG using RIPA, BIPA, T-TAS, and EMSA (data not shown).

In addition, caplacizumab was used in the clinical study and approved as a bivalent nanobody which has two

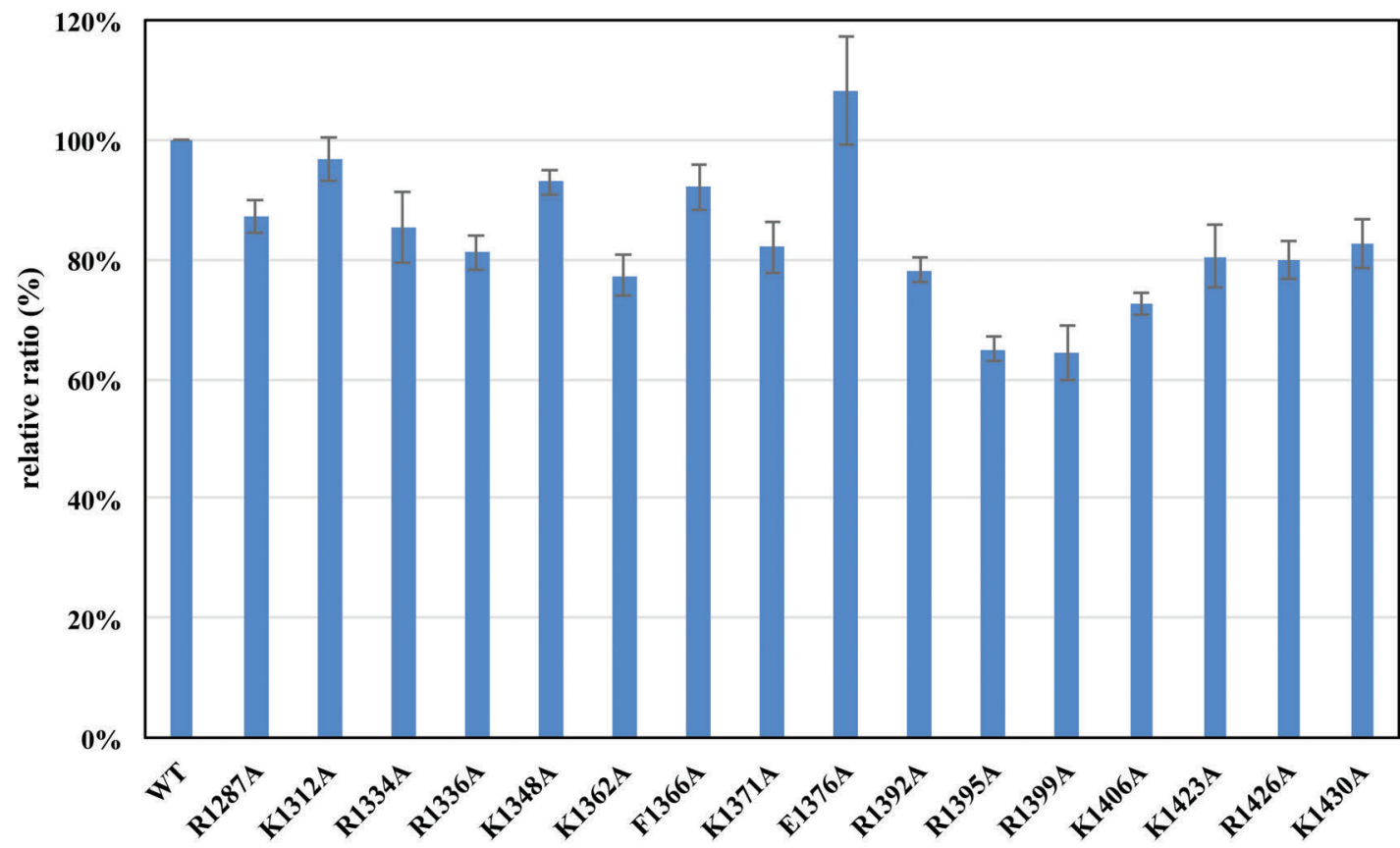

Figure 5. Analysis of caplacizumab binding sites to the von Willebrand factor A1 domain using alanine-scanning mutants with surface plasmon resonance. We performed surface plasmon resonance (SPR) analysis to investigate caplacizumab binding sites to the von Willebrand factor (VWF) A1 domain. After immobilizing caplacizumab on the sensor chip CM5, 16 alanine-substituted VWF A1 mutants were analyzed. The relative binding volume of wild-type (WT) VWF A1 was defined as $100 \%$. The binding amount of each mutant was expressed as a ratio relative to WT. Five mutants were considered to bind to caplacizumab based on having a relative ratio above the cutoff of $80 \%$. These results indicate that these five amino acids (K1362, R1392, R1395, R1399, and K1406) in the VWF A1 domain were important in caplacizumab binding.

\section{TAGX-0004}

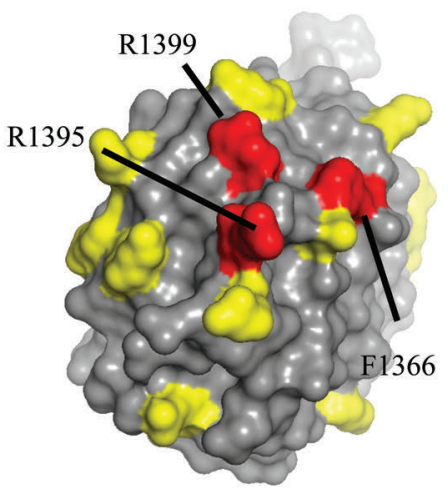

$\underline{\mathrm{ARC1779}}$

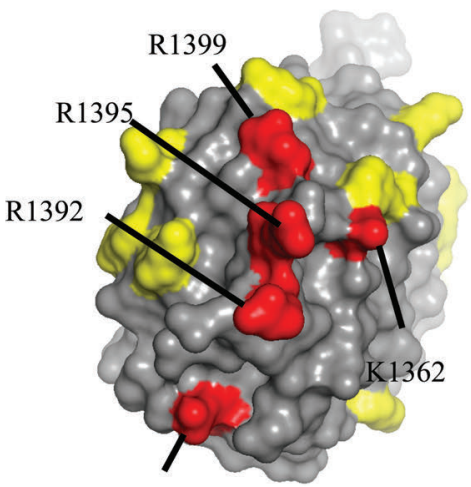

R1287
Caplacizumab

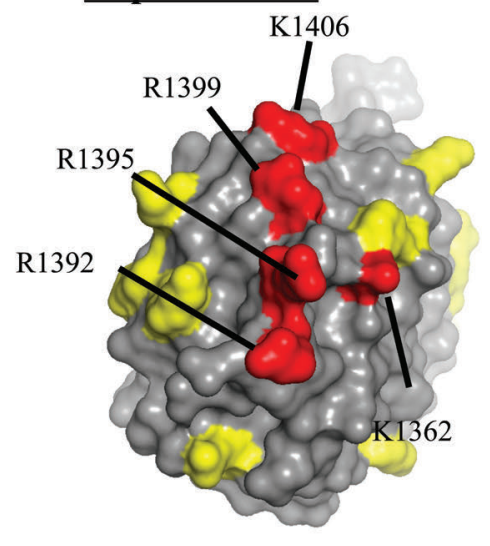

Figure 6. Von Willebrand factor A1 domain binding sites to TAGX-0004, ARC1779, and caplacizumab. Amino acids colored yellow did not contribute to von Willebrand factor (VWF) A1 binding to aptamers or caplacizumab. Amino acids colored in red were necessary for binding to aptamers or caplacizumab. Three amino acids (F1366, R1395, and R1399) in the VWF A1 domain were identified as TAGX-0004 binding sites. Five amino acids (R1287, K1362, R1392, R1395, and R1399) were important for binding to ARC1779. Finally, five amino acids (K1362, R1392, R1395, R1399, and K1406) were important for binding to caplacizumab. 
binding sites to the target. On the other hand, two aptamers used in this study were formed as monovalent. It is known that the direct comparison of an affinity of monovalent entity with an avidity of bivalent entity is not straightforward. Nevertheless, it is intriguing that TAGX0004 has shown a comparable inhibitory effect against caplacizumab in the studies we performed, and how the bivalent form of TAGX-0004, which is not available though, behaves in the same experiments.

Treatment with caplacizumab has demonstrated a rapid decrease of VWF ristocetin cofactor assay in patients with aTTP and the levels of VWF antigen and factor VIII were also transiently reduced with caplacizumab treatment compared with placebo due to an increased clearance of the caplacizumab-VWF complex. ${ }^{11}$ Whether or not TAGX0004 demonstrates an equivalent effect is still to be studied using an in vivo model. Nevertheless, judging from the binding ability of TAGX-0004 to plasma-derived human VWF confirmed with EMSA (data not shown), it is likely that the aptamer shows a similar effect as caplacizumab in aTTP patients.

In conclusion, we confirmed the potency of TAGX-0004 to prevent platelet thrombus formation in vitro. Epitope mapping of the binding sites of TAGX-0004 compared with ARC1779 and caplacizumab provided us with information on each molecule's efficacy and characteristics. Caplacizumab is now coming into use as a first-line therapy for aTTP. However, there are still challenges to be overcome with this agent, such as bleeding adverse events and high cost. ${ }^{31}$ TAGX-0004 has the potential to overcome these problems and could be developed as a promising drug not only for aTTP, but also for various VWF-mediated thrombotic disorders such as acute coronary syndrome and cerebral infarction.

\section{Funding}

This work was supported by research grants from the Ministry of Health, Labour, and Welfare of Japan.

\section{References}

1. Sadler JE. Biochemistry and genetics of von Willebrand factor. Annu Rev Biochem. 1998;67:395-424.

2. Ruggeri ZM. Von Willebrand factor, platelets and endothelial cell interactions. J Thromb Haemost. 2003;1(7):1335-1342.

3. Montalescot G, Philippe F, Ankri A, et al. Early increase of von Willebrand factor predicts adverse outcome in unstable coronary artery disease: beneficial effects of enoxaparin. French Investigators of the ESSENCE Trial. Circulation. 1998;98(4):294-299.

4. Ray KK, Morrow DA, Gibson CM, Murphy S, Antman EM, Braunwald E. Predictors of the rise in vWF after ST elevation myocardial infarction: implications for treatment strategies and clinical outcome: An ENTIRE-TIMI 23 substudy. Eur Heart J. 2005;26(5):440-446.

5. Bath PM, Blann A, Smith N, Butterworth RJ. Von Willebrand factor, P-selectin and fibrinogen levels in patients with acute ischaemic and haemorrhagic stroke, and their relationship with stroke sub-type and functional outcome. Platelets. 1998;9(34):155-159

6. Moake JL, Rudy CK, Troll JH, et al. Unusually large plasma factor VIII:von Willebrand factor multimers in chronic relapsing thrombotic thrombocytopenic purpura. N Engl J Med. 1982;307(23):14321435.

7. Gragnano F, Sperlongano S, Golia E, et al. The role of von Willebrand factor in vascular inflammation: from pathogenesis to targeted therapy. Mediators Inflamm. 2017;2017:5620314.

8. Siller-Matula JM, Krumphuber J, Jilma B. Pharmacokinetic, pharmacodynamic and clinical profile of novel antiplatelet drugs targeting vascular diseases. Br J Pharmacol. 2010;159(3):502-517.

9. Bae ON. Targeting von Willebrand factor as a novel anti-platelet therapy; application of ARC1779, an Anti-vWF aptamer, against thrombotic risk. Arch Pharm Res. 2012; 35(10):1693-1699.

10. Matsui T, Hori A, Hamako J, et al. Mutant botrocetin-2 inhibits von Willebrand factorinduced platelet agglutination. J Thromb Haemost. 2017;15(3):538-548.

11. Peyvandi F, Scully M, Kremer Hovinga JA, et al. Caplacizumab for acquired thrombotic thrombocytopenic purpura. N Engl Med. 2016;374(6):511-522

12. Scully M, Cataland SR, Peyvandi F, et al. Caplacizumab treatment for acquired thrombotic thrombocytopenic purpura. N Engl J Med. 2019;380(4):335-346.

13. Cataland SR, Peyvandi F, Mannucci PM, et al. Initial experience from a double-blind, placebo-controlled, clinical outcome study of ARC1779 in patients with thrombotic thrombocytopenic purpura. Am J Hematol. 2012;87(4):430-432.

14. Matsunaga KI, Kimoto M, Hirao I. Highaffinity DNA aptamer generation targeting von Willebrand factor A1-domain by genetic alphabet expansion for systematic evolution of ligands by exponential enrichment using two types of libraries composed of five different bases. J Am Chem Soc. 2017;139(1):324-334.

15. Hosokawa K, Ohnishi T, Kondo T, et al. A novel automated microchip flow-chamber system to quantitatively evaluate thrombus formation and antithrombotic agents under blood flow conditions. J Thromb Haemost. 2011;9(10):2029-2037.

16. Hirao I, Kimoto M, Mitsui $T$, et al. An unnatural hydrophobic base pair system: site-specific incorporation of nucleotide analogs into DNA and RNA. Nat Methods. 2006;3(9):729-735

17. Yamashige R, Kimoto M, Takezawa Y, et al Highly specific unnatural base pair systems as a third base pair for PCR amplification. Nucleic Acids Res. 2012;40(6):2793-2806.

18. Diener JL, Daniel Lagasse HA Duerschmied D, et al. Inhibition of von Willebrand factor-mediated platelet activation and thrombosis by the anti-von Willebrand factor A1-domain aptamer ARC1779. J Thromb Haemost. 2009; 7(7):1155-1162

19. Huizinga EG, Tsuji S, Romijn RA, et al. Structures of glycoprotein Ibalpha and its complex with von Willebrand factor A1 domain. Science. 2002;297(5584):1176 1179

20. Matsushita T, Meyer D, Sadler JE. Localization of von willebrand factor-binding sites for platelet glycoprotein $\mathrm{Ib}$ and botrocetin by charged-to-alanine scanning mutagenesis. J Biol Chem. 2000;275(15): 11044-11049.
21. Huang RH, Fremont DH, Diener JL, Schaub RG, Sadler JE. A structural explanation for the antithrombotic activity of ARC1172, a DNA aptamer that binds von Willebrand factor domain A1. Structure. 2009;17(11): 1476-1484.

22. Furlan M, Robles R, Galbusera M, et al. von Willebrand factor-cleaving protease in thrombotic thrombocytopenic purpura and the hemolytic-uremic syndrome. N Engl J Med. 1998:339(22):1578-1584.

23. Tsai HM, Lian EC. Antibodies to von Willebrand factor-cleaving protease in acute thrombotic thrombocytopenic purpura. N Engl J Med. 1998;339(22):15851594.

24. Gragoudas ES, Adamis AP, Cunningham ET, Jr., Feinsod M, Guyer DR, Group VISiONCT. Pegaptanib for neovascular age-related macular degeneration. $\mathrm{N}$ Engl J Med. 2004;351(27):2805-2816.

25. Gomez-Outes A, Suarez-Gea ML, Lecumberri R, Rocha E, Pozo-Hernandez C, Vargas-Castrillon E. New parenteral anticoagulants in development. Ther Adv Cardiovasc Dis. 2011;5(1):33-59.

26. Vavalle JP, Cohen MG. The REG1 anticoagulation system: a novel actively controlled factor IX inhibitor using RNA aptamer technology for treatment of acute coronary syndrome. Future Cardiol. 2012;8(3):371382.

27. Ponce AT, Hong KL. A mini-review: clinical development and potential of aptamers for thrombotic events treatment and monitoring. Biomedicines. 2019;7(3):55.

28. Gilbert JC, DeFeo-Fraulini T, Hutabarat RM, et al. First-in-human evaluation of anti von Willebrand factor therapeutic aptamer ARC1779 in healthy volunteers. Circulation. 2007;116(23):2678-2686.

29. Nimjee SM, White RR, Becker RC, Sullenger BA. Aptamers as therapeutics. Annu Rev Pharmacol Toxicol. 2017; 6(57):61-79

30. Chen Y, Roberts JR, Orje JN, et al Identification of a VWFA1 mutation attenuating thrombus growth but not platelet adhesion. Blood. 2017;130(Suppl_1):547.

31. Mazepa MA, Masias C, Chaturvedi S. How targeted therapy disrupts the treatment paradigm for acquired TTP - the risks, benefits and unknowns. Blood. 2019;134(5): 415-420. 